\title{
Evaluation of pharmacodynamic properties and safety of Cinnamomum Zeylanicum (ceylon cinnamon) in health adults: study protocol for a phase I clinical trial
}

\begin{abstract}
Purpose: In-vitro and in-vivo studies in animals suggests that Ceylon Cinnamon has anti-microbial, anti-parasitic, anti-oxidant and free radical scavenging properties. This article summarizes the study protocol for a Phase-I clinical trial in humans.

Methods: The study will be conducted as a phase-I clinical trial for a period of 3 months at the University of Colombo, Sri Lanka. A total of 30 healthy adults will be recruited for the study. The treatment drug is a capsule containing Cinnamomum zeylanicum as the active ingredient. The subjects will receive cinnamon capsules (85mg, $250 \mathrm{mg}$ and $500 \mathrm{mg}$ ) daily for a period of 3 months, in monthly escalating doses. The visits and the evaluations will be done as follows: screening, 1 month, 2 months and 3 month. The following primary and secondary outcome measures will be evaluated: FBG, total cholesterol, triglycerides, LDL cholesterol and HDL cholesterol, appetite, blood pressure, anthropometry, dietary assessment, liver profile, renal profile, $\mathrm{FBC}$ and urinalysis.
\end{abstract}

Findings/implications: We describe the protocol for a clinical trial design evaluating the efficacy and safety Ceylon cinnamon. The result of the present study, positive or negative, should provide a step change in the evidence guiding current and future policies regarding the use of cinnamon dietary supplementation.

Trial registration: Sri Lanka Clinical Trials Registry (SLCTR/2013/001).

Keywords: Cinnamomum zeylanicum, ceylon cinnamon, efficacy, pharmacodynamics, safety, adverse effects, sri lanka, adults
Volume 3 Issue 2 - 2017

\author{
Priyanga Ranasinghe,' Ranil Jayawardena, ${ }^{2}$ GA \\ Sirimal Premakumara, ${ }^{3}$ Prasad Katulanda, ${ }^{4}$ \\ Godwin R Constantine, ${ }^{4}$ Priyadarshani \\ Galappaththy' \\ 'Department of Pharmacology, University of Colombo, Sri Lanka \\ 2Department of Physiology, University of Colombo, Sri Lanka \\ ${ }^{3}$ Industrial Technology Institute, Colombo, Sri Lanka \\ ${ }^{4}$ Department of Clinical Medicine, University of Colombo, Sri \\ Lanka
}

Correspondence: Priyanga Ranasinghe, Department of Pharmacology, Faculty of Medicine, University of Colombo, Sri Lanka, Tel 0094 7I4 0394I3, Fax 00940112 596368, Email priyanga.ranasinghe@gmail.com

Received: October 29, 2016 | Published: March 10, 2017
Abbreviations: ALD, alcoholic liver disease; FBG, fasting blood glucose; LDL, low density lipoprotein; HDL, high density lipoprotein; VAS, visual analog scales; FBC, full blood count; SBP, systolic blood pressure; DBP, diastolic blood pressure; BMI, body mass index; WC, waist circumference; HC, hip circumference; WHR, waist hip ratio; FFQ, food frequency questionnaire; CRF, case record form; ALT, alanine aminotransferase; AST, aspartate aminotransferase; HOMA-IR, homeostasis model of assessment-insulin resistance; CRF, case report form; SOP, standard operating procedure; CRA, clinical research associate; SPSS, statistical package for the social sciences; WHO, world health organization; AUC, area under the curve

\section{Introduction}

Cinnamon, the inner bark of a tropical evergreen tree has two main varieties, Cinnamomum cassia (Cinnamomum aromaticum) and Cinnamomum zeylanicum. In native Ayurvedic medicine Cinnamon is considered a remedy for respiratory, digestive and gynecological ailments. Sri Lanka produces the largest quantity and the best quality cinnamon. Cinnamomum zeylanicum, also known as Ceylon cinnamon (the source of its Latin name, zeylanicum) or 'true cinnamon' is indigenous to Sri Lanka. On the basis of the appearance of the quill, it is possible to differentiate Ceylon cinnamon from the others. ${ }^{1}$ It is softer and lighter in color and rolled in layers whereas the others are darker, hard, and hollow and rolled in only one layer. ${ }^{1}$ Different oils obtained from this interesting plant yield different constituents: Cinnamaldehyde (in the bark oil), eugenol (in the leaf oil), and camphor (in the root-bark oil). ${ }^{2}$ The fruits and flowers are a rich source of transcinnamyl acetate. ${ }^{3}$ A recent review by Ranasinghe et al. ${ }^{4}$ summarized the most recent evidence on medicinal benefits of Ceylon Cinnamon and showed that it has anti-microbial, anti-parasitic, anti-oxidant and free radical scavenging properties. ${ }^{4}$ In addition the authors concluded that $\mathrm{CZ}$ seems to lower blood glucose, serum cholesterol and blood pressure, suggesting beneficial cardiovascular effects. ${ }^{4}$

In-vitro studies and few in-vivo animal studies have evaluated the mechanism for these medicinal benefits of Ceylon Cinnamon. The high dietary fibre content of could result in reduced intestinal lipid absorption, and the high vitamin/anti-oxidant is likely to result in increased lipid metabolism. ${ }^{5}$ Insulin plays a key role in lipid metabolism and it is possible that increased serum Insulin levels following Ceylon Cinnamon administration also contributes towards reducing lipid levels. ${ }^{6}$ Results of studies in animals have indicated that cinnamon regulates blood pressure levels through peripheral vasodilatation. ${ }^{7}$ This vasodilatation might be partially through $\mathrm{Ca}^{2+}$ channels blocking properties. ${ }^{8}$ Cinnamon extracts are known to increase Tristetraprolin mRNA and protein levels, Tristetraprolins have anti-inflammatory effects due to destabilizing of proinflammatory mRNA. ${ }^{9}$ This could be the reason for the anti-inflammatory actions observed. The antimicrobial action is considered to arise mainly from the potential of 
hydrophobic essential oils to disrupt the bacterial cell membrane and its structures which leads to ion leakage. ${ }^{10}$ Trans-cinnamaldehyde is also known to inhibits bacterial acetyl-CoA carboxylase. ${ }^{11}$ The different parts of the Ceylon Cinnamon plant possess the same array of hydrocarbons in varying proportions, and this chemical diversity is likely to be the reason for the wide-variety of medicinal benefits observed. $^{4}$

Another important difference between 'true' cinnamon and the cassia cinnamon is their coumarin content. ${ }^{12}$ The coumarin content in Ceylon cinnamon appears to be very small to cause health risks, whereas the coumarin level in Cinnamomum aromaticum appears to be much higher and may pose health risks if consumed in higher quantity on a regular basis. ${ }^{12,13}$ The usage of Cassia cinnamon as a regular supplement with meals was not advocated or the daily dosage was restricted in many countries due to the toxic effects of Cinnamomum aromaticum on the liver and coagulation. ${ }^{12}$ In contrast Cinnamomum zeylanicum has shown to contain a lesser content of coumarin ${ }^{12,14}$ and thus it may be possible that Ceylon cinnamon could be used in higher doses without toxic effects for longer durations.

The absence of properly conducted randomized controlled human trials is a barrier to decide on efficacy and safety of long term Cinnamon use in humans and to determine whether these effects have public health implications. Animal studies on Ceylon cinnamon have not demonstrated any significant adverse effects or toxicity on the liver, kidney and the pancreas. ${ }^{15,16}$ However, it is necessary to conduct safety studies in humans, prior to advocating regular use of Ceylon cinnamon for any medicinal benefit. This article summarizes the study protocol for a Phase I clinical trial in humans, looking at the efficacy and safety of Ceylon Cinnamon in healthy adults.

\section{Methods}

\section{Objectives}

To evaluate the pharmacodynamic properties and the safety of Cinnamomum zeylanicum extract in healthy adults.

\section{Study design}

The study will be conducted at the Faculty of Medicine, University of Colombo, Sri Lanka as a phase I clinical trial for a period of 3 month. It will be assessing the efficacy and safety of daily supplementation of capsules containing Cinnamomum zeylanicum in healthy adults.

\section{Sample size}

The Phase-I clinical trial will be conducted with 30 healthy volunteers, for a period of 3 months. Subject will be recruited on a voluntary basis by open advertisement (attached herewith). Subjects will receive a Cinnamomum zeylanicum containing capsule daily for a period of 3 months.

\section{Population}

Participants will be recruited on a voluntarily basis after open advertisement. Informed written consent will be obtained from all study participants. Details of inclusion and exclusion criteria are given below.

\section{Inclusion and exclusion criteria}

Inclusion criteria: Healthy adults between $18-50$ years of age.

\section{Exclusion criteria}

a. Alcohol consumption $>20 \mathrm{~g} /$ day, patients with Alcoholic Liver Disease (ALD), patients with cirrhosis and abnormal baseline liver, renal function tests or blood counts.

b. Patient with any chronic illness.

c. Patient on any long term medication including any nutritional supplements.

d. Lactation, pregnancy or unwillingness to use an effective form of birth control for women of child bearing years.

e. Any condition in the opinion of the primary investigator that would contraindicate the patient's participation.

\section{Suspension criteria}

a. Subject's demand to discontinue the study.

b. Serious adverse events or unusual changes in clinical test results.

c. Principal investigator's decision to terminate the study (low rates of compliance, complications, or unable to sustain the study for various reasons).

\section{Interventions}

The treatment drug is a capsule containing Cinnamomum zeylanicum as the active ingredient. Raw cinnamon was obtained from a single cinnamon cultivator (to maintain uniformity) in Galle district of Sri Lanka. This raw cinnamon powder was refined in batches, according to the process described below. The preparation of the refined formulation was done at the Industrial Technology Institute laboratory, Colombo, Sri Lanka. The dried powder of Cinnamomum zeylanicum (Ceylon cinnamon) will be extracted from stem barks to distilled water using Soxlet apparatus and the resulting hot water extract will be freeze-dried to obtain crude water extract from which the capsules will be prepared. The subjects will be prescribed Cinnamomum zeylanicum capsules for a period of 3 months. To evaluate the safety of Cinnamomum zeylanicum 3 doses of refined cinnamon would be used (85mg, 250mg and 500mg). These doses correspond to $1 \mathrm{~g}, 3 \mathrm{~g}$ and $6 \mathrm{~g}$ of crude cinnamon, which has shown beneficial health properties in cassia cinnamon in previous research studies. Each person would be given $85 \mathrm{mg}$ daily for 30 days during the 1 st month (each capsule $85 \mathrm{mg}$ ), $250 \mathrm{mg}$ daily for 30 days during the 2 nd month (each capsule $250 \mathrm{mg}$ ) and $500 \mathrm{mg}$ daily for 30 days during the $3 \mathrm{rd}$ month ( 2 capsules of $250 \mathrm{mg}$ per day). Drug compliance of patients would be evaluated by pill counting (number of pill's returned monthly).

\section{Study period}

The study will be for a period of 3 month and the visits and the evaluations will be done as follows; screening (visit 0), 1 month (visit 1), 2 months (visit 2) and 3 months (visit 3/final visit).

\section{Outcomes}

Primary outcome index: The following biochemical assessment will be done at baseline, at the stated intervals and on completion of the study (Table 1); FBG, total cholesterol, triglycerides, LDL cholesterol and HDL cholesterol. Furthermore, appetite will be evaluated using Visual Analog Scales (VAS). The following information will be recor- 
ded/measured for the safety assessment; vital signs, general medical examinations, Full Blood Count (FBC), renal function test, liver function test, urinalysis and adverse events.

\section{Secondary outcome index}

a. Measurement of systolic (SBP) and diastolic blood pressure (DBP).

b. Anthropometric assessment such as body weight, height, Body Mass Index (BMI), waist circumference (WC), hip circumference (HC) and Waist Hip Ratio (WHR).

c. Dietary assessment using a validated Food Frequency Questionnaire (FFQ). ${ }^{17}$

Table I Summarized study schedule at each visit in the clinical trial

\begin{tabular}{|c|c|c|c|c|}
\hline & $\begin{array}{l}\text { Visit } 0 \\
\text { (screening } \\
\text { visit) }\end{array}$ & $\begin{array}{l}\text { Visit I } \\
\text { (I } \\
\text { month) }\end{array}$ & $\begin{array}{l}\text { Visit } 2 \\
(2 \\
\text { months) }\end{array}$ & $\begin{array}{l}\text { Visit } 3 \\
(3 \\
\text { month) }\end{array}$ \\
\hline $\begin{array}{l}\text { Medical history } \\
\text { taking }\end{array}$ & $\cdot$ & - & $\cdot$ & - \\
\hline FBG & $\cdot$ & $\cdot$ & $\cdot$ & $\cdot$ \\
\hline Lipid profile' & - & & & - \\
\hline Liver function ${ }^{2}$ & $\cdot$ & $\cdot$ & $\cdot$ & $\cdot$ \\
\hline Renal function ${ }^{3}$ & - & - & $\cdot$ & - \\
\hline $\mathrm{FBC}^{4}$ & $\cdot$ & & & $\cdot$ \\
\hline Blood pressure & $\cdot$ & $\cdot$ & $\cdot$ & $\cdot$ \\
\hline $\begin{array}{l}\text { Physical } \\
\text { examination }\end{array}$ & $\cdot$ & · & - & . \\
\hline Urinanalysis ${ }^{6}$ & - & & & - \\
\hline Electrocardiogram & $\cdot$ & & & $\cdot$ \\
\hline FFQ/VAS & - & · & - & - \\
\hline
\end{tabular}

'Total cholesterol, triglyceride, HDL cholesterol

${ }^{2} \mathrm{AST}, \mathrm{ALT}$, total bilirubin, PT/INR

${ }^{3}$ Creatinine, blood urea nitrogen (BUN)

${ }^{4} \mathrm{WBC}, \mathrm{RBC}$, haemoglobin (HGB), hematocrit (HCT), platelet count (PLT)

${ }^{5}$ Body weight $(\mathrm{kg})$, height $(\mathrm{cm})$, waist circumference $(\mathrm{cm})$, hip circumference $(\mathrm{cm})$, waist hip ratio

${ }^{6}$ Urine microscopy, urine for sugar and protein

\section{Procedures}

\section{Recruitment}

Patients will be recruited on a voluntarily basis from open advertisement. Those who consent to participate in the trial will visit the trial site voluntarily.

\section{Study schedule}

The details of items which will be measured at every visit are described in Table 1.

\section{Measurement tools}

Anthropometric measurements: Body weight will be measured using a calibrated electronic floor scale (SECA 815 by SECA GmbH \& Co. Kg. Hamburg, Germany) to the nearest $0.1 \mathrm{~kg}$. Height will be measured to the nearest $0.1 \mathrm{~cm}$ using an upright plastic portable Stadiometer (SECA 217 by SECA GmbH \& Co. Kg. Hamburg, Germany). BMI will be calculated as weight (in kilograms) divided by the square of height (in meters). Waist circumference (WC) will be measured with a non-elastic tape (SECA 203 by SECA GmbH \& Co. $\mathrm{Kg}$. Hamburg, Germany) at a point midway between the lower border of the rib cage and the iliac crest at the end of normal expiration. Similarly, the hip circumference also will be measured at widest part of the buttocks in the inter-trochantric level to the nearest $0.1 \mathrm{~cm}$. All anthropometric measurements will be made by using standard equipment and following WHO guidelines.

Dietary measurements: A culturally validated FFQ will be used to obtain habitual intake of calorie, macro-nutrients and micronutrients. ${ }^{17} \mathrm{~A}$ VAS, $10 \mathrm{~cm}$ in length with words anchored at each end, expressing the most positive and the most negative rating, will be used to assess hunger, satiety, fullness and desire to eat.

\section{Compliance calculation}

Subjects are asked to return remaining drugs and their compliance will be evaluated by using the formula given below,

Compliance $(\%)=[($ distributed drugs-remaining drugs $) /$ distributed drugs $] \times 100$

\section{Statistical analysis}

Parametric and non parametric statistical tests will be applied using the SPSS version 14 (SPSS Inc., Chicago, IL, USA) and Stata/SE 10.0 (Stata Corporation, College Station, TX, USA) for the data analysis. Data will be entered by a minimum number of dedicated staff and saved in a dedicated computer with password protection. For each of the outcomes, multilevel regression analysis will be used to examine differences between trial arms. For binary outcomes the model will be logistic and for continuous outcomes the model will be linear regression. All analyses will follow intention-to-treat principles and a pre-specified analysis plan. Where appropriate, sensitivity analyses will be conducted (for example, control for additional covariates; and bootstrapped $\mathrm{p}$ values for skewed outcomes). In the case of missing data values, we will apply mean imputation and regression imputation where rates are low, and consider multiple imputations where they exceed $10 \%$.

\section{Adverse effect evaluation and management}

In the event of a probable adverse reaction the following precautions would ensure timely identification and management of patients;

a. Reporting: Mechanisms would be setup to ensure direct reporting of probable adverse events to investigators by patients (via telephone available 24-hour on all days).

b. During follow up visits probable adverse events would be noted by history and examination and investigated upon in detail. All Adverse Effects observed will be documented in the Case Record Form (CRF).

c. All serious adverse events will be reported to the Ethics Review Committee, Faculty of Medicine, University of Colombo and National Pharmaco-vigilance Unit in Department of Pharmacology, Faculty of Medicine, university of Colombo. 
d. A Data Safety Monitoring Board (DSMB) identified by the investigators will evaluate all the adverse events at regular intervals

e. Investigations: Liver and renal function, and FBC would be assessed as detailed above.

f. Termination of study: In the event of major adverse effects occurring in a significant proportion of the study population the study would be terminated pending further investigation.

\section{Data collection}

After filling out the CRF (case report form), data collection will be performed according to the standard operating procedures (SOPs), by the trained clinical research associates (CRAs).

\section{Data management and monitoring}

Storage: Data will be entered by a minimum number of dedicated staff and saved in a dedicated computer with password protection. Samples would be stored in a secure facility, with redundant measures to ensure specimens are kept in compliant conditions at all times when in storage. Expert staff that has been trained specifically in sample storage and transportation would ensure all regulatory issues are properly handled. Storage technologies with the capability of monitoring the temperature of samples around the clock would be utilized.

Sample disposal: After each analysis is completed and with the approval of the Principal Investigator, the samples stored in the storage facility may be disposed of by the sample custodian. A Sample Disposal Sheet will be completed and kept for further reference.

\section{Ethical approval}

The study has been approved by the Ethics Review Committee of Faculty of Medicine, University of Colombo, Sri Lanka (EC/12/157). The trial is also registered at the Sri Lanka Clinical Trials Registry (SLCTR/2013/001). The study will be conducted in compliance with the Declaration of Helsinki and the Good Clinical Practice (GCP) guidelines.

\section{Discussion}

In this article we describe the protocol for a phase 1 clinical trial design evaluating the efficacy and safety of Cinnamomum zeylanicum (Ceylon cinnamon) in healthy adults. To our knowledge this is one of the first trials evaluating the effects of medium-long term supplementation of Ceylon cinnamon in humans. Hence, the present study will provide the required foundation regarding safety for future large-scale multi-centered clinical trials evaluating various medicinal properties of Ceylon cinnamon. Given the present interest in using a variety of herbal/nutritional supplements to treat patients, properly planned and methodical scientific studies are a timely necessity. However, in relation to cinnamon presently there are no welldesigned trials conducted for a satisfactory time period to support/ refute claims regarding safety. Hence the result of the present study, positive or negative, should provide a step change in the evidence guiding current and future policies regarding the use of cinnamon dietary supplementation.

\section{Trial status}

Ongoing

\section{Competing interest}

The author(s) declare that they have no competing interests.

\section{Funding}

This work is supported by a grant from the University of Colombo (AP/03/2012/CG/08).

\section{Acknowledgements}

None.

\section{Conflict of interest}

The author declares no conflict of interest.

\section{References}

1. Kawatra P, Rajagopalan R. Cinnamon: Mystic powers of a minute ingredient. Pharmacognosy Res. 2015;7(Suppl 1):1-6.

2. Wijesekera RO. Historical overview of the cinnamon industry. CRC Crit Rev Food Sci Nutr. 1978;10(1):1-30.

3. Jayaprakasha GK, Rao LJ. Chemistry, biogenesis, and biological activities of Cinnamomum zeylanicum. Crit Rev Food Sci Nutr. 2011;51(6):547-562

4. Ranasinghe P, Pigera S, Premakumara GA, et al. Medicinal properties of 'true' cinnamon (Cinnamomum zeylanicum): a systematic review. $B M C$ Complement Altern Med. 2013;13:275.

5. Lattimer JM, Haub MD. Effects of dietary fiber and its components on metabolic health. Nutrients. 2010;2(12):1266-1289.

6. Ranasinghe P, Jayawardana R, Galappaththy P, et al. Efficacy and safety of 'true' cinnamon (Cinnamomum zeylanicum) as a pharmaceutical agent in diabetes: a systematic review and meta-analysis. Diabet Med. 2012;29(12):1480-1492

7. Preuss HG, Echard B, Polansky MM, et al. Whole cinnamon and aqueous extracts ameliorate sucrose-induced blood pressure elevations in spontaneously hypertensive rats. J Am Coll Nutr. 2006;25(2):144-150.

8. Wansi SL, Nyadjeu P, Ngamga D, et al. Blood pressure lowering effect of the ethanol extract from the stembark of cinnamomum zeylanicum (lauraceae) in rats. Pharmacol online. 2007;3:166-176.

9. Cao H, Urban JF, Anderson RA. Cinnamon polyphenol extract affects immune responses by regulating anti-and proinflammatory and glucose transporter gene expression in mouse macrophages. $J$ Nutr. 2008;138(5):833-840.

10. Rana IS, Singh A, Gwal R. In vitro study of antibacterial activity of aromatic and medicinal plants essential oils with special reference to cinnamon oil. Int J Pharm Pharm Sci. 2011;3(4):376-380.

11. Meades G, Henken RL, Waldrop GL, et al. Constituents of cinnamon inhibit bacterial acetyl CoA carboxylase. Planta Med. 2010;76(14):15701575 .

12. Lungarini S, Aureli F, Coni E. Coumarin and cinnamaldehyde in cinnamon marketed in Italy: a natural chemical hazard? Food Addit Contam Part A Chem Anal Control Expo Risk Assess. 2008;25(11):1297-1305.

13. Abraham K, Wohrlin F, Lindtner O, et al. Toxicology and risk assessment of coumarin: focus on human data. Mol Nutr Food Res. 2010;54(2):228 239.

14. Rychlik M. Quantification of free coumarin and its liberation from glucosylated precursors by stable isotope dilution assays based on liquid chromatography-tandem mass spectrometric detection. J Agric Food Chem. 2008;56(3):796-801. 
15. Ranasinghe P, Perera S, Gunatilake M, et al. Effects of Cinnamomum zeylanicum (Ceylon cinnamon) on blood glucose and lipids in a diabetic and healthy rat model. Pharmacognosy Res. 2012;4(2):73-79.

16. Beejmohun V, Peytavy Izard M, Mignon C, et al. Acute effect of Ceylon cinnamon extract on postprandial glycemia: alpha-amylase inhibition, starch tolerance test in rats, and randomized crossover clinical trial in healthy volunteers. BMC Complement Altern Med. 2014;14:351.
17. Jayawardena R, Swaminathan S, Byrne NM, et al. Development of a food frequency questionnaire for Sri Lankan adults. Nutr J. 2012;11(1):63. 